\title{
A Hamiltonian formalism for bosonic membranes
}

\author{
B.P. Dolan \\ Department of Mathematical Physics, St. Patrick's College, Maynooth, Ireland, and School of Theoretical Physics, Dublin Institute for \\ Advanced Studies, 10 Burlington Road, Dublin 4, Ireland
}

Received 5 June 1989; in revised form 22 September 1989

\begin{abstract}
An action for bosonic membranes, which has no cosmological constant, is studied. The Hamiltonian formalism is developed, with a view to quantisation, using Dirac's method for constrained systems. The commutators of the independent canonical variables are evaluated in a co-ordinate gauge, at least to lowest order in $\hbar$.
\end{abstract}

\section{Introduction}

There has recently been much interest in membranes as a generalisation of strings in the extended object approach to fundamental physics [1-3]. The purpose of this paper is to explore the properties of an action for bosonic membranes, first proposed in [4]. This action differs from the more usual actions used for membranes in that it is polynomial in the dynamical fields (quartic plus quadratic) and does not require a cosmological constant. It is based on a generalisation of a conformally invariant $\sigma$ model action in four dimensions developed in [5].

The motivation for studying this action comes from an analogue in string theory where there is a choice of classically equivalent Lagrangians, the NambuGoto form [6,7] or the $\sigma$ model from [8]. Although these give the same classical theory, the quantum theories differ, the latter offering more flexibility in terms of the critical dimension [9]. It therefore seems plausible that different classical actions for the membrane may lead to differing quantum theories.

To quantise this $\sigma$ model membrane action, the Hamiltonian formalism is adopted and constraints are handled using Dirac's formalism. The Poisson algebra of the constraints is evaluated and first and second class constraints are identified. The first class constraints are transformed into second class constraints by choosing a gauge which eliminates all the longitudinal degrees of freedom, leaving only transverse co-ordinates as free variables. The Dirac brackets for the independent canonical variables are evaluated, at least to lowest order in $\hbar$, and are shown to be the same as the Poisson brackets, thus providing a convenient starting point for the quantum theory.

\section{The embedding}

A bosonic membrane embedded in a $D$ dimensional space-time, $(\mathscr{M}, g)$, with a $D$ dimensional metric, $g$, sweeps out a three dimensional world volume, $\Sigma$, (two space and one time dimension). $x^{\mu}$ will denote coordinates in $D$ dimensions $(\mu, v, \ldots=0,1, \ldots, D-1)$ and $g_{\mu \nu}$ the metric components. Co-ordinates on the world volume of the membrane will be denoted by $\sigma^{a}$ $(a, b, \ldots=0,1,2)$. The embedding, $x^{\mu}(\sigma)$, of the world volume of the membrane into space-time induces a metric on $\Sigma$,

$G_{a b}(\sigma)=\partial_{a} x^{\mu}(\sigma) \partial_{b} x^{v}(\sigma) g_{\mu v}(x(\sigma))$

and a three dimensional connection (everything is assumed torsion free)

$$
\begin{aligned}
\gamma_{b c}^{a} & =\frac{1}{2} G^{a d}\left(G_{d b, c}+G_{d c, b}-G_{b c, d}\right) \\
& =G^{a d} \partial_{d} x^{\rho}\left(\partial_{b} \partial_{c} x^{\mu}+\partial_{b} x^{v} \partial_{c} x^{\tau} \Gamma_{v \tau}^{\mu}\right) g_{\rho \mu}
\end{aligned}
$$

where $\Gamma_{v \rho}^{\mu}$ is the $D$ dimensional Christoffel connection $\Gamma_{v \rho}^{\mu}=\frac{1}{2} g^{\mu \tau}\left(g_{\tau v, \rho}+g_{\tau \rho, v}-g_{v \rho, \tau}\right)$

and $G^{a b}$ is the inverse of $G_{a b}$. (There is a slight abuse of notation here, since $\Gamma_{v \rho}^{\mu}$ means $\Gamma_{v \rho}^{\mu}(x(\sigma))$ i.e. the Christoffel connection on $(\mathscr{M}, g)$ restricted to $\Sigma$ which is, of course, distinct from $\gamma_{b c}^{a}$.)

Co-variant differentiation of tensors, $T^{\mu}$, on $\mathscr{M}$ will be denoted by; thus

$T_{; v}^{\mu}=T_{, v}^{\mu}+\Gamma_{v \rho}^{\mu} T^{\rho}$

while co-variant differentiation of tensors on $\Sigma$ will be denoted by $\|$ thus

$T_{\| b}^{a}=T_{, b}^{a}+\gamma_{b c}^{a} T^{c}$.

A useful object in the sequel will be the projection 
operator

$P_{\tau}^{\mu}(x(\sigma))=G^{a b} \partial_{a} x^{v} \partial_{b} x^{\mu} g_{v \tau}$

which has the property that

$P_{\tau}^{\mu} P_{v}^{\tau}=P_{v}^{\mu}$

It is not difficult to prove the following identity for any smooth embedding

$P_{v}^{\mu} \square x^{v}=-P_{v}^{\mu} P^{\rho \tau} \Gamma_{\rho \tau}^{v}$

where

$\square x^{\mu}=G^{a b}\left(\partial_{a} x^{\mu}\right)_{\| b}$.

\section{The action}

To determine the dynamics we introduce an independent metric on $\Sigma, H_{a b}(\sigma)$, which is a priori unrelated to $G_{a b}(\sigma) . H_{a b}(\sigma)$ will later be identified with $G_{a b}(\sigma)$ only through the dynamics. Denote the inverse of $H_{a b}$ by $H^{a b}$.

The action is taken to be

$L=\int_{\Sigma} d^{3} \sigma \mathscr{L}(\sigma)$

with

$$
\begin{aligned}
\mathscr{L}(\sigma)= & -\sqrt{-\operatorname{det} H}\left(H^{a b} G_{a b}\right. \\
& \left.+\frac{1}{2} H^{a b} H^{c d}\left(G_{a b} G_{c d}-G_{a d} G_{b c}\right)\right)
\end{aligned}
$$

where the integral is over the world volume of the membrane. The dynamical variables are $H^{a b}(\sigma)$ and $x^{\mu}(\sigma)$, the latter appearing in the definition of $G^{a b}(\sigma),(1)$.

It was reported in [4] that this action is classically equivalent to the $\mathrm{Nambu}-$ Goto action $[6,7]$,

$L=-6 \int_{\Sigma} d^{3} \sigma \sqrt{-\operatorname{det} G}$

with the dynamical relation

$G^{a b}=H^{a b}$.

A proof is given in the next section.

The introduction of the independent metric, $H_{a b}$, is similar in spirit to the course followed in [8] for the bosonic string Lagrangian. The Lagrangian (11) is one of a class of Lagrangians introduced in [4] with a view to avoiding the introduction of a cosmological constant into the dynamics.

\section{Lagrangian formalism}

To determine the dynamics of (11), first vary $H^{a b}$ in $\mathscr{L}$ to give

$$
\begin{aligned}
\frac{\delta L}{\delta H^{a b}}= & \frac{1}{2} \sqrt{-\operatorname{det} H} H_{a b} \\
& \cdot\left(H^{c d} G_{c d}+\frac{1}{2} H^{f g} H^{c d}\left(G_{f g} G_{c d}-G_{f c} G_{g d}\right)\right) \\
& -\sqrt{-\operatorname{det} H}\left(G_{a b}+H^{c d}\left(G_{a b} G_{c d}-G_{a c} G_{b d}\right)\right) \\
= & 0 .
\end{aligned}
$$

Define a matrix $A_{b}^{a}=H^{a c} G_{c b}$, then this is equivalent to $\left(A-\mathbf{1}_{3 \times 3}\right)\left(A-(\operatorname{tr} A) \mathbf{1}_{3 \times 3}\right)$.

To solve this equation for $A$, note that any square matrix can be put into Jordan normal form by a similarity transformation [10]. Thus $A$ can have one of three forms,

$$
\begin{aligned}
& A=S^{-1}\left(\begin{array}{ccc}
a_{1} & 0 & 0 \\
1 & a_{1} & 0 \\
0 & 1 & a_{1}
\end{array}\right) S \quad A=S^{-1}\left(\begin{array}{ccc}
a_{1} & 0 & 0 \\
1 & a_{1} & 0 \\
0 & 0 & a_{2}
\end{array}\right) S \\
& A=S^{-1}\left(\begin{array}{ccc}
a_{1} & 0 & 0 \\
0 & a_{2} & 0 \\
0 & 0 & a_{3}
\end{array}\right) S
\end{aligned}
$$

It is not difficult to show that the first two forms cannot satisfy (15), hence only the last form is a possible solution. Since $A$ satisfies a quadratic equation, (15), there are only two possible values for $a_{1}, a_{2}$ and $a_{3}$. By a suitable relabelling, the only possible combinations are; either $a_{1}=a_{2}=a_{3}=1$, or $a_{1}=a_{2}=-1$ and $a_{3}=+1$. We reject the second solution since it admits the possibility of $H^{a b}$ having a different signature from $G^{a b}$. This is most easily seen in the Euclidean sector where we can always find a co-ordinate system $\sigma^{a}$ so that $G^{a b}=\delta^{a b}$ at any given point (though not elsewhere, in general). Then $\operatorname{det} H=(\operatorname{det} A)^{-1} \operatorname{det} G$ implies that the second solution must give an $H$ with a negative eigenvalue. In the Lorentzian sector the argument is not so clear cut. e.g. $G^{a b}=\operatorname{diag}(-1,+1,+1)$ and $S=1$ gives $H^{a b}=\operatorname{diag}(+1,-1,+1)$ with the second form of $A$. This $H$ has the same signature as $G$, but a different causal structure. If we demand, as seems physically reasonable, that $H$ has the same causal structure as $G$ then only the first form of $A$ is allowed. Hence the only physically acceptable solution is $A=\mathbf{1}$, which gives

$H^{a b}=G^{a b}$.

Putting this back into (11) reproduces the NambuGoto action (12). Varying $x^{\mu}$ now leads to the Lagrangian equations of motion

$\square x^{\mu}=-P^{v \rho} \Gamma_{\nu \rho}^{\mu}$

with $P^{\mu v}$ given in terms of $x^{\mu}$, by (6) and (1). This is, of course, a dynamical equation and as such is more restrictive than the identity (8), though it is obviously compatible with (8). Essentially, the identity (8) says that the dynamics is all in the transverse part of (18).

\section{Hamiltonian formalism}

The first step towards quantisation of this system is to construct the Hamiltonian, using $\sigma^{0}=\tau$ as the time variable. Rather than using $H^{a b}$ as dynamical variables, it is convenient (as is usual with co-ordinate independent systems [11]) to use lapse and shift functions, $N$ and $N^{i}$, together with the two dimensional space-like 
metric $h^{i j},(i, j=1,2)$, defined by

$h_{i j}=H_{i j} \quad H^{i j}=h^{i j}+N^{i} N^{j} / N^{2}$

$h_{i j} N^{j}=H_{0 i} \quad N^{i} / N^{2}=H^{0_{i}}$

$H_{00}=-N^{2}+h_{i j} N^{i} N^{j} \quad H^{00}=-1 / N^{2}$

$\sqrt{-\operatorname{det} H}=N \sqrt{\operatorname{det} h} \equiv N \sqrt{h}$.

The canonical momenta conjugate to $x^{\mu}, N, N^{i}$ and $h^{i j}$ are

$\pi_{\mu}=\frac{\delta L}{\delta \dot{x}^{\mu}} \quad K=\frac{\delta L}{\delta \dot{N}} \quad K_{i}=\frac{\delta L}{\delta \dot{N}^{i}} \quad K_{i j}=\frac{\delta L}{\delta \dot{h}^{i j}}$.

Immediately there are six primary constraints $[11,12]$

$K \approx 0 \quad K_{i} \approx 0 \quad K_{i j} \approx 0$

( $\approx$ means weakly zero, as defined in [12]).

Thus, the momenta conjugate to $N, N^{i}$ and $h^{i j}$ vanish and we find (suppressing repeated space-time indices) that the canonical Hamiltonian is

$$
\begin{aligned}
H_{c}(\tau)= & \int_{r_{(\tau)}} d^{2} \sigma\left(\frac{N}{2 \sqrt{h}}\left(\pi M^{-1} \pi\right)\right. \\
& \left.+\frac{N \sqrt{h}}{4}\left(\partial_{i} x M \partial_{j} x\right) h^{i j}+N^{i}\left(\pi \partial_{i} x\right)\right)
\end{aligned}
$$

where

$M_{\mu \nu}=2\left(G_{i j} g_{\mu \nu}-\partial_{i} x^{\rho} \partial_{j} x^{\tau} g_{\rho \mu} g_{\tau v}\right) h^{i j}+2 g_{\mu \nu}$

with $G_{i j}$ given by (1). $r(\tau)$ is the two dimensional space-like surface of constant $\tau$, embedded in $\Sigma$, and $d^{2} \sigma=d \sigma^{1} d \sigma^{2}$.

Any linear combination of the primary constraints can be added to (22) without affecting the dynamics. Thus

$$
\tilde{H}(\tau)=H_{c}(\tau)+\int_{r(\tau)} d^{2} \sigma\left(u K+u^{i} K_{i}+u^{i j} K_{i j}\right)
$$

where $u, u^{i}$ and $u^{i j}$ are arbitrary functions of the canonical variables, is an equally good Hamiltonian. $\widetilde{H}(\tau)$ is termed the effective Hamiltonian in [11] and the total Hamiltonian in [12].

Immediately we see that, in passing to the quantum theory, there will be problems with the operator ordering due to the fact that $M$ contains $x^{\mu}$, and so will not commute with $\pi_{\mu}$. One possible approach is to Weyl order and replace $\pi_{\mu}\left(M^{-1}\right)^{\mu \nu} \pi_{\nu}$ with $(1 / 2)\left(\pi_{\mu} \pi_{v}\left(M^{-1}\right)^{\mu v}+\left(M^{-1}\right)^{\mu v} \pi_{\mu} \pi_{v}\right)$, but this eventually proves inadequate. For the moment the best that can be done, without getting into horrendous algebraic complexities, is to say that any extra terms introduced into the calculation by the non-commutativity of $\pi_{\mu}$ and $x^{\mu}$ in the quantum theory will involve extra powers of $\hbar$, and so we get at least a first approximation to the quantum theory by ignoring them.

Following Dirac, [12], we must demand that the time evolution of the constraints (21) vanish weakly for consistency

$$
\begin{aligned}
& \dot{K}(\sigma)=\{K(\sigma), \tilde{H}\}=-\frac{\delta \tilde{H}}{\delta N(\sigma)} \approx-\frac{\delta H_{c}}{\delta N(\sigma)} \approx 0 \\
& \dot{K}_{i}(\sigma) \approx-\frac{\delta H_{c}}{\delta N^{i}(\sigma)} \approx 0 \quad \dot{K}_{i j}(\sigma) \approx-\frac{\delta H^{c}}{\delta h^{i j}(\sigma)} \approx 0 .
\end{aligned}
$$

$(\{$,$\} denote Poisson brackets.)$

We find the following six secondary constraints

$$
\begin{aligned}
\chi(\sigma)= & \frac{1}{2 \sqrt{h}}\left(\pi M^{-1} \pi\right)+\frac{\sqrt{h}}{4}\left(\partial_{i} x M \partial_{j} x\right) h^{i j} \approx 0 \\
\chi_{i}(\sigma)= & \left(\pi \partial_{i} x\right) \approx 0 \\
\chi_{i j}(\sigma)= & \frac{N}{4}\left(\frac{\sqrt{h}}{2}\left(\partial_{k} x M \partial_{l} x\right) h^{k l}-\frac{1}{\sqrt{h}}\left(\pi M^{-1} \pi\right)\right) h_{i j} \\
& +\frac{N}{\sqrt{h}}\left(\pi M^{-1}\right)^{\mu} M_{i j, \mu v}\left(M^{-1} \pi\right)^{\nu} \\
& -\frac{N}{2} \sqrt{h}\left(\partial_{i} x M \partial_{j} x\right) \\
\approx & 0
\end{aligned}
$$

where

$M_{i j, \mu v}=G_{i j} g_{\mu v}-\partial_{i} x^{\rho} \partial_{j} x^{\tau} g_{\rho \mu} g_{\tau v}$

The secondary constraints

$\chi \approx 0 \quad \chi_{i} \approx 0 \quad \chi_{i j} \approx 0$

are classically equivalent to the algebraic Lagrangian equations of motion $G_{a b}=H_{a b}$ and so, as operator equations in the quantum theory, they are equivalent, at least to lowest order in $\hbar$.

Note that

$H_{c}(\tau)=\int_{\gamma_{(\tau)}} d^{2} \sigma\left(N \chi+N^{i} \chi_{i}\right)$

is weakly vanishing, as expected on general grounds [12].

Demanding that the time evolution of the $\chi$ 's vanish weakly gives no new constraints, but merely determines the $u$ 's in (24).

In the quantum theory, the distinction between primary and secondary constraints cases to be important, and a more relevant classification is that of first and second class constraints. The Poisson brackets of first class constraints give rise to linear combinations of first class constraints and are weakly vanishing, whereas the Poisson brackets of second class constraints do not vanish, even weakly.

Calculating the Poisson brackets of the twelve constraints $K$ 's and $\chi$ 's) with each other (and making liberal use of the Lagrangian version of the constraints, (13), after the evaluation of their Poisson brackets) gives the following weakly non-zero brackets (after some tedious algebra)

$$
\begin{aligned}
& \left\{K_{i j}(\sigma), \chi_{k l}\left(\sigma^{\prime}\right)\right\} \\
& \quad \approx N \sqrt{h}\left(h_{i k} h_{j l}+h_{i l} h_{j k}-\frac{2}{3} h_{i j} h_{k l}\right) \delta^{(2)}\left(\sigma-\sigma^{\prime}\right)
\end{aligned}
$$




$$
\begin{aligned}
& \left\{\chi(\sigma), \chi_{i j}\left(\sigma^{\prime}\right)\right\} \\
& \quad \approx \frac{2}{3} N \pi_{\mu}\left(\frac{1}{3} X_{k l}^{\mu} h^{k l} h_{i j}-X_{i j}^{\mu}\right) \delta^{(2)}\left(\sigma-\sigma^{\prime}\right) \\
& \left\{\chi_{i j}(\sigma), \chi_{k l}\left(\sigma^{\prime}\right)\right\} \\
& \approx-\left(N^{2} / 9\right) \pi_{\mu}\left(X_{i j}^{\mu} h_{k l}-X_{k l}^{\mu} h_{i j}\right) \delta^{(2)}\left(\sigma-\sigma^{\prime}\right) \\
& \left\{\chi_{i j}(\sigma), \chi_{k}\left(\sigma^{\prime}\right)\right\} \\
& \approx-2 N \sqrt{h}\left(h_{i k} \partial_{j} \delta^{(2)}\left(\sigma-\sigma^{\prime}\right)\right. \\
& \left.\quad+h_{j k} \partial_{i} \delta^{(2)}\left(\sigma-\sigma^{\prime}\right)-(2 / 3) h_{i j} \partial_{k} \delta^{(2)}\left(\sigma-\sigma^{\prime}\right)\right) \\
& \quad-2 N \sqrt{h}\left(\partial_{k} h_{i j}-\frac{1}{3 h}\left(\partial_{k} h\right) h_{i j}\right) \delta^{(2)}\left(\sigma-\sigma^{\prime}\right)
\end{aligned}
$$

where $X_{i j}^{\mu} \equiv \partial_{i} \partial_{j} x^{\mu}+\partial_{i} x^{v} \partial_{j} x^{\rho} \Gamma_{v \rho}^{\mu}, h=\operatorname{det}\left(h_{i j}\right), \partial_{i}=$ $\partial / \partial \sigma^{i}$, and all functions on the right hand sides are evaluated at $\sigma$. All other Poisson brackets among the constraints are weakly vanishing. Note in passing that it makes no difference whether the ordinary, $\left(\partial_{i}\right)$, or covariant, $(\| i)$, derivative is used on $\partial_{j} x^{\mu}$ in the definition of $X_{i j}^{\mu}$ above since these are weakly equivalent when contracted with $\pi_{\mu}$

$\pi_{\mu}\left(\partial_{j} x^{\mu}\right)_{\| i}=\pi_{\mu}\left(\partial_{i} \partial_{j} x^{\mu}-\gamma_{i j}^{k} \partial_{k} x^{\mu}\right) \approx \pi_{\mu} \partial_{i} \partial_{j} x^{\mu}$.

However, the term involving $\Gamma_{v \rho}^{\mu}$ cannot be omitted in the definition of $X_{i j}^{\mu}$.

It is still necessary to disentangle which linear combinations of constraints are first class and which are second class. Following [13], define

$$
\begin{aligned}
& \tilde{\chi}=\chi+\left(\frac{1}{3 \sqrt{h}}\right) K_{i j} X^{i j, \mu} \tau_{\mu} \\
& \tilde{\chi}_{i}=\chi_{i}+K_{j k} \partial_{i} h^{j k}+2 \partial_{k}\left(K_{i j} h^{j k}\right) \\
& \tilde{\chi}_{i j}=\chi_{i j}-\left(\frac{N}{18 \sqrt{h}}\right) h_{i j} K_{k l} X^{k l, \mu} \pi_{\mu}
\end{aligned}
$$

(indices $i, j, k$ and $l$ are raised with the two dimensional metric, $h^{i j}$ ). and

With these definitions $K, K_{i}, \tilde{\chi}$ and $\tilde{\chi}_{i}$ are first class,

$$
\begin{aligned}
\left\{K_{i j}(\sigma), \tilde{\chi}_{k l}\left(\sigma^{\prime}\right)\right\} \approx & N \sqrt{h}\left(h_{i k} h_{j l}+h_{i l} h_{j k}\right. \\
& \left.-(2 / 3) h_{i j} h_{k l}\right) \delta^{(2)}\left(\sigma-\sigma^{\prime}\right) .
\end{aligned}
$$

It is convenient to eliminate the term with $-\frac{2}{3}$, by defining

$$
\chi_{k l}^{*}=\tilde{\chi}_{k l}+\left(h^{i j} \tilde{\chi}_{i j}\right) h_{k l}
$$

giving

$$
\begin{aligned}
\left\{K_{i j}(\sigma), \chi_{k l}^{*}\left(\sigma^{\prime}\right)\right\} & \approx N \sqrt{h}\left(h_{i k} h_{j l}+h_{i l} h_{j k}\right) \delta^{(2)}\left(\sigma-\sigma^{\prime}\right) \\
& \equiv C_{i j, k l}\left(\sigma, \sigma^{\prime}\right) .
\end{aligned}
$$

Apart from the infinite rank due to $\sigma, C_{i j, k l}$ is an antisymmetric $6 \times 6$ matrix. It has an inverse

$\left(C^{-1}\right)^{i j, k l}\left(\sigma, \sigma^{\prime}\right)=\frac{1}{4 N \sqrt{h}}\left(h^{i k} h^{j l}+h^{i l} h^{j k}\right) \delta^{(2)}\left(\sigma-\sigma^{\prime}\right)$ so that

$$
\begin{array}{r}
\int_{r(\tau)} d^{2} \sigma^{\prime \prime} C_{i j, k l}\left(\sigma, \sigma^{\prime \prime}\right)\left(C^{-1}\right)^{k l, i^{\prime} j^{\prime}}\left(\sigma^{\prime \prime}, \sigma^{\prime}\right) \\
=(1 / 2)\left(\delta_{i}^{i^{\prime}} \delta_{j}^{j^{\prime}}+\delta_{i}^{j^{\prime}} \delta_{j}^{i^{\prime}}\right) \delta^{(2)}\left(\sigma-\sigma^{\prime}\right) .
\end{array}
$$

\section{Gauge conditions}

To proceed, the first class constraints must be eliminated by suitable gauge choices, making all constraints second class, [11]. The obvious choices $\dot{N}=0$ and $N^{i}=0$, effectively eliminate $K$ and $K_{i}$, leaving only $\tilde{\chi}$ and $\tilde{\chi}_{i}$ as first class constraints. A further three constraints (gauge condition) on $x^{\mu}$ and $\pi_{\mu}$ are required to turn $\tilde{\chi}$ and $\tilde{\chi}_{i}$ into second class constraints. The authors of [13] follow the string theorists and fix the center of mass and momentum of the membrane, in the light cone gauge. While these two gauge conditions fix the gauge uniquely for string, they are not sufficient for membranes. The third degree of freedom is left unfixed in [13]. We shail choose a different gauge, the 'co-ordinate gauge'.

$x^{0}=\tau \quad x^{1}=\sigma^{1} \quad x^{2}=\sigma^{2}$

leaving only the transverse co-ordinates, $x^{m}, m=3,4, \ldots$, $D-1$ as degrees of freedom. The three constraints $\tilde{\chi}$ and $\tilde{\chi}_{i}$ can now be used to eliminate $\pi_{0}, \pi_{1}$ and $\pi_{2}$.

A related, but not identical, gauge has been investigated for strings, [14]. There the gauge used was $x^{0}=\tau, \pi_{0}=$ const (only two gauge fixing conditions are required for the string).

We must be careful that the co-ordinates $\tau, \sigma^{1}$ and $\sigma^{2}$ are nowhere singular on $\Sigma$. It may be necessary to choose more than one co-ordinate patch on $\Sigma$ and then match them together on the overlaps using a gauge transformation. These problems can be avoided by restricting ourselves to world volumes, $\Sigma$, which can be covered by a single co-ordinate patch. e.g. an open membrane with $-\infty<\tau<\infty, 0 \leqq \sigma^{1}, \sigma^{2} \leqq \pi$ with topology $\mathbb{R} \times[0, \pi] \times[0, \pi]$ (though the metric on $\sigma$ need not be flat). However, this restriction to contractible $\Sigma$ does not seem to be necessary, provided suitable gauge transformations are used on the coordinate overlaps.

First write the gauge conditions as constraints

$\phi^{0}=x^{0}-\tau \quad \phi^{1}=x^{1}-\sigma^{1} \quad \phi^{2}=x^{2}-\sigma^{2}$

then this gauge is enforced by imposing the conditions

$\phi^{a} \approx 0 \quad(a=0,1,2)$.

The matrix of Poisson brackets of constraints, $C$, must now be enlarged to include $\phi^{a}$. We need

$$
\begin{aligned}
\left\{\phi^{a}(\sigma), \phi^{b}\left(\sigma^{\prime}\right)\right\} & \approx 0 \\
\left\{\phi^{a}(\sigma), \tilde{\chi}\left(\sigma^{\prime}\right)\right\} & \approx \frac{1}{6 \sqrt{h}} \pi_{\mu} g^{\mu a} \delta^{(2)}\left(\sigma-\sigma^{\prime}\right) \\
\left\{\phi^{a}(\sigma), \tilde{\chi}_{j}\left(\sigma^{\prime}\right)\right\} & \approx \delta_{j}^{a} \delta^{(2)}\left(\sigma-\sigma^{\prime}\right) \\
\left\{\phi^{a}(\sigma), \chi_{i j}^{*}\left(\sigma^{\prime}\right)\right\} & \approx-\frac{1}{12 \sqrt{h}} h_{i j} \pi_{\mu} g^{\mu a} \delta^{(2)}\left(\sigma-\sigma^{\prime}\right) .
\end{aligned}
$$


$C$ now becomes an antisymmetric $12 \times 12$ matrix $\left(s, s^{\prime}=1, \ldots, 12\right)$

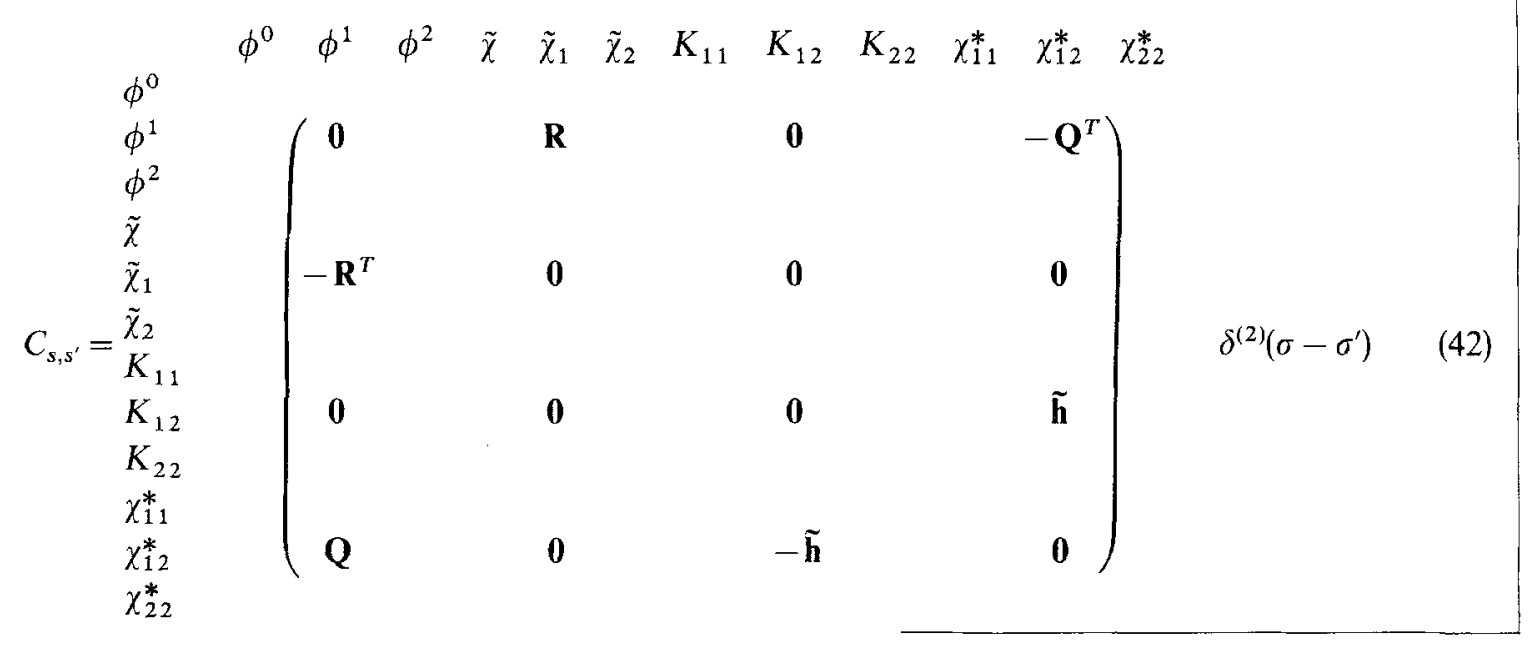

where the $3 \times 3$ matrices $\mathbf{R}, \mathbf{Q}$ and $\tilde{\mathbf{h}}$ are defined as

$\mathbf{R}=\left(\begin{array}{lll}\pi^{0} /(6 \sqrt{h}) & 0 & 0 \\ \pi^{1} /(6 \sqrt{h}) & 1 & 0 \\ \pi^{2} /(6 \sqrt{h}) & 0 & 1\end{array}\right)$

$\mathbf{Q}_{i j,}{ }^{a}=\frac{1}{12 \sqrt{h}}\left(h_{i j} \pi_{\mu} g^{\mu a}\right)$ and $\tilde{\mathbf{h}}_{i j, k l}=\sqrt{h}\left(h_{i k} h_{j l}+h_{i l} h_{j k}\right)$.

For example

$C_{1,4},\left(\sigma, \sigma^{\prime}\right)=\frac{\pi^{0}}{6 \sqrt{h}} \delta^{(2)}\left(\sigma-\sigma^{\prime}\right) \approx\left\{\phi^{0}(\sigma), \tilde{\chi}\left(\sigma^{\prime}\right)\right\}$.

The inverse matrix $\left(C^{-1}\right)^{s, s^{\prime}}$ is readily obtained

$$
\begin{aligned}
& \left(C^{-1}\right)^{s, s^{\prime}}\left(\sigma, \sigma^{\prime}\right) \\
& \quad=\left(\begin{array}{cccc}
\mathbf{0} & -\left(\mathbf{R}^{T}\right)^{-1} & \mathbf{0} & \mathbf{0} \\
\mathbf{R}^{-1} & \mathbf{0} & \mathbf{S} & \mathbf{0} \\
\mathbf{0} & -\mathbf{S}^{T} & \mathbf{0} & -\tilde{\mathbf{h}}^{-1} \\
\mathbf{0} & \mathbf{0} & \tilde{\mathbf{h}}^{-1} & \mathbf{0}
\end{array}\right) \delta^{(2)}\left(\sigma-\sigma^{\prime}\right)
\end{aligned}
$$

with

$$
\begin{aligned}
& \mathbf{R}^{-1}=\left(\begin{array}{ccc}
(6 \sqrt{h}) / \pi^{0} & 0 & 0 \\
-\left(\pi^{1} / \pi^{0}\right) & 1 & 0 \\
-\left(\pi^{2} / \pi^{0}\right) & 0 & 1
\end{array}\right) \\
& \mathbf{S}=\left(\begin{array}{ccc}
h^{11} /(4 \sqrt{h}) & h^{12} /(4 \sqrt{h}) & h^{22} /(4 \sqrt{h}) \\
0 & 0 & 0 \\
0 & 0 & 0
\end{array}\right)
\end{aligned}
$$

and

$$
\left(\tilde{\mathbf{h}}^{-1}\right)^{i j, k l}=\frac{1}{4 \sqrt{h}}\left(h^{i k} h^{j l}+h^{i l} h^{j k}\right) .
$$

The commutators of the remaining canonical vari- ables, $x^{m}$ and $\pi_{m}$, in the quantum theory are now obtained from the Dirac brackets, [12]

$$
\begin{aligned}
\left\{A(\sigma), B\left(\sigma^{\prime}\right)\right\}^{*} & \\
= & \left\{A(\sigma), B\left(\sigma^{\prime}\right)\right\}-\iint_{r^{(\tau)}} d^{2} \sigma_{1} d^{2} \sigma_{2}\left\{A(\sigma), \phi_{s}\left(\sigma_{1}\right)\right\} \\
& \cdot\left(C^{-1}\right)^{s, s^{\prime}}\left(\sigma_{1}, \sigma_{2}\right)\left\{\phi_{s^{\prime}}\left(\sigma_{2}\right), B\left(\sigma^{\prime}\right)\right\}
\end{aligned}
$$

where $\phi_{s}$ represent the twelve second class constraints.

We find quite simply

$\left\{x^{m}(\sigma), \pi_{n}\left(\sigma^{\prime}\right)\right\}^{*} \approx \delta_{n}^{m} \delta^{(2)}\left(\sigma-\sigma^{\prime}\right)$

i.e. the degrees of freedom transverse to the world volume of the membrane are unaffected by the presence of the constraints.

Of course, the Dirac brackets of $\pi_{a}$ and $x^{a}$ will be more complicated, but these are no longer free canonical variables, due to the constraints $\phi^{a}, \tilde{\chi}$ and $\tilde{\chi}_{i}$. Explicitly

$$
\begin{array}{lll}
\phi^{a} & \text { and } \quad \tilde{\chi}_{1} \approx 0 \Rightarrow \pi_{1} \approx-\pi_{m} \partial_{1} x^{m} \\
\phi^{a} & \text { and } \quad \tilde{\chi}_{2} \approx 0 \Rightarrow \pi_{2} \approx-\pi_{m} \partial_{2} x^{m}
\end{array}
$$

and $\tilde{\chi} \approx 0$ can be used to find an expression for $\pi_{0}$ in terms of $x^{m}$ and $\pi_{m}$, but we do not write down because it is not very illuminating.

Finally, the extended Hamiltonian, [12], is obtained by adding linear combinations of $\left(K, K_{i}, \tilde{\chi}, \tilde{\chi}_{i}\right) \equiv\left(\psi_{A}\right)$ to (24)

$$
H_{E}(\tau)=\tilde{H}(\tau)+\int_{\gamma_{(\tau)}} d^{2} \sigma v^{A}(\sigma) \psi_{A}(\sigma) \quad A=1, \ldots, 6 .
$$

The functions $v^{A}$ are determined by demanding consistent time evolution of the gauge constraints.

\section{Conclusions}

Dirac brackets have been evaluated for the Hamiltonian (22), arising from the action (11), for a membrane moving in $D$ dimensions, at least to lowest order in $\hbar$. 
The co-ordinate gauge,

$x^{0}=\tau \quad x^{1}=\sigma^{1} \quad x^{2}=\sigma^{2}$

has been used, with the canonical degrees of freedom $\left\{x^{m}, \pi_{n}\right\}(m, n=3,4, \ldots, D)$. The Dirac brackets are

$\left\{x^{m}(\sigma), \pi_{n}\left(\sigma^{\prime}\right)\right\}^{*}=\delta_{n}^{m} \delta^{(2)}\left(\sigma-\sigma^{\prime}\right)$

at least to lowest order in $\hbar$.

Classically the Lagrangian (11) is equivalent to the Nambu-Goto Lagrangian (12) and so the above analysis of Dirac brackets for the Hamiltonian (22) would lead to the same classical physics as that of the Hamiltonian following from (12). However, as explained in the introduction, one would expect the quantum theories to differ. In particular, when $(\mathscr{M}, g)$ is $D$ dimensional Minkowski space, manifest Lorentz invariance is lost with the choice of gauge (52), and one must check that the theory is still Lorentz invariant. For the string this requirement constrains $D$ to be 26 , using the Nambu-Goto action $[14,15]$, but with the $\sigma$ model action there is the possibility that $D$ can be less than 26. The intrinsic non-linearity of the membrane makes an analogous calculation much more difficult here. There is some evidence that there is a critical dimension of 27 for the bosonic membrane, using the Nambu-Goto action (12), [16], and one can hope that this could be relaxed to $D$ less than 27 using (11) but this subject merits further study.
Acknowledgement. I am grateful to Ed Corrigan for bringing my attention to [14].

\section{References}

1. J. Hughes, J. Liu, J. Polchinski: Phys. Lett. 180B (1986) 370

2. A. Achúcarro, J.M. Evans, P.K. Townsend, D.L. Wiltshire: Phys. Lett. 198B (1987) 441

3. E. Bergshoeff, E. Sezgin, P.K. Townsend: Phys. Lett. 189B (1987) 75

4. B.P. Dolan, D.H. Tchrakian: Phys. Lett. 202B (1988) 211

5. B. Felsager, J.M. Leinaas: Phys. Lett. 94B (1980) 192; Ann. Phys. $130(1980) 461$

6. Y. Nambu: Lectures at the Copenhagen Summer Symposium, (1970)

7. T. Goto: Prog. Theor. Phys. 46 (1971) 1560

8. L. Brink, P. DiVecchia, P.S. Howe: Phys. Lett. 56B (1976) 471; S. Deser, B. Zumino, Phys. Lett. 65B (1976) 369

9. A.M. Polyakov: Phys. Lett. 103B (1981) 207

10. V.I. Smirnov: A course in higher mathematics. Vol. III, Part 1. Oxford, New York: Pergamon 1964.

11. A. Hanson, T. Regge, C. Teitelboim: Constrained Hamiltonian systems. Accademia dei Lincei, Roma (1976)

12. P.A.M. Dirac: Lectures on quantum mechanics. Belford Graduate School of Science. Yeshiva University, New York (1964)

13. E. Bergshoeff, E. Sezgin, Y. Tani: Nucl. Phys. B298 (1988) 187

14. P. Goddard, A.J. Hanson, G. Ponzano: Nucl. Phys. B89 (1987) 76

15. P. Goddard, J. Goldstone, C. Rebbi, C.B. Throne: Nucl. Phys. B56 (1973) 109

16. U. Marquard, M. Scholl: Phys. Lett. 209B (1988) 434 